\title{
Computational Intelligence Based Technique for Multi-DG Installation in Transmission System
}

\author{
Muhamad Fazrizal Faiz Sahilahudin ${ }^{1}$, Ismail Musirin ${ }^{1}$, Shahrizal Jelani ${ }^{2, *}$ and Mohd Helmi Mansor ${ }^{3}$ \\ ${ }^{1}$ Faculty of Electrical Engineering, Universiti Teknologi MARA, 40000 Shah Alam, Selangor, Malaysia \\ ${ }^{2}$ Faculty of Engineering, Technology \& Built Environment, UCSI University, 1, Jalan Puncak Menara Gading, Taman Connaught, \\ 56000 Kuala Lumpur, Malaysia \\ ${ }^{3}$ Department of Electrical \& Electronics, College of Engineering, Universiti Tenaga Nasional, Jalan IKRAM-UNITEN, 43000 Kajang, \\ Selangor, Malaysia
}

\begin{abstract}
The increasing electricity demand in transmission system has caused the power transmission system experiencing stress condition. This phenomenon has forced the system to need urgent additional supply to maintain system adequacy, in particular the de-regulated power system environment. Distributed generation (DG) has been identified as one of the possible solutions to address this issue. DG installation has the capability to reduce transmission loss and improve the voltage profile. This paper presents evolutionary programming (EP) technique for optimizing the sizing and locations in DG installation. In this study, several DGs have been installed to address the voltage profile improvement and loss minimization; implemented on the IEEE 30-Bus Reliability Test System (RTS). Results obtained from the study revealed that, installation of multi-DGs in a transmission system has significantly minimized the transmission loss along with voltage profile improvement.
\end{abstract}

\section{Introduction}

Electricity demand in most countries gradually increased in line with the country development. Nevertheless, the existing infrastructure may not able to meet the demand due to the increasing crude oil; also affected by the depreciation of the generation stations in the grid system. Thus, compensation efforts have been identified as the possible remedial action to alleviate between the demand and supply. One of suitable options is the compensation scheme contributed by the installation of distributed generation (DG) in the transmission and distribution [1]. DG can be defined as electric power generation within the distribution or on the customer side of the network [2]. In other words, DG acts as an additional supply to the system to cater the demand increment [3]. Among the DG types are small gas turbines, micro-turbines, fuel cells, wind and solar energy, biomass and small hydropower [4]-[5]. The types of DG commonly used on power networks is the wind energy with the injected power below 50MW. There are many levels of DG in the network ranging from micro range to the large one in the form of MW. The small range is between $(1 \mathrm{~W}-5 \mathrm{~kW})$ and $(5 \mathrm{~kW}-5 \mathrm{MW})$; followed by the medium range (5 MW-50 MW), and lastly the large one which is (50 MW-300 MW) [6]. As mention before medium range is commonly used in the system for installation DG. The installation of DG in a transmission and a distribution network may give effects either positive or negative effect[7]. The positive effect resulted by the installation of DG is line loss reduction and improvement of voltage profile [6]-[7]. For example, DG can balance up the local power demand in both the transmission and distribution sectors [8]. With the recent technology advancement, installation of DG in the power distribution

and transmission system can provide economical solution and keep network working properly. This will require optimization process to ensure the optimal or correct location to locate the DG in power system [9]. Improper or non-optimal sizing or locations may lead to over-compensation or under-compensation to the system [10-12]. Various optimization techniques have been proposed to solve optimization problems such as the DG installation scheme. Some of the popular techniques are such as Genetic Algorithm (GA), Particle Swarm Optimization (PSO), Evolutionary Programming (EP) and Ant Colony Optimization (ACO). Other important method is the improved analytical (IA) method [13]. In [14] the improved tabu search algorithm was used to improve the local search ability and to reduce computation time as well as to minimize the line losses in transmission and distribution systems. Other reliable approach is the adaptive cuckoo [15] and PSO [16] search for optimal location of DG in distribution network. In this paper, the Evolutionary Programming (EP) as a computational intelligence technique was used to determine the optimal location and sizing of distributed generation in transmission system. EP is a productive technique in which its functionally for various search, classification and optimization problems is quite

Corresponding author: shahrizal@ucsiuniversity.edu.my 
outstanding. The objective of the optimization is to minimize the losses in the network and improving the voltage profile in transmission system. Results obtained from the study, validated on the IEEE 30-Bus Reliability Test System is promising and has the feasibility for larger and bulk power system implementation.

\section{Problem Formulation}

The objectives of this study are loss minimization and maximization of voltage profile. EP is used to minimize the power losses as well to maximize the voltage profile which require random numbers to represent the location and sizing of the DG. These random numbers will subject to several constraint equations.

The inequality constraint on voltage of each bus is given by:

$$
|V i| \min \leq V i \leq|V i| \max
$$

Inequality constraints for the sizing of DG is subject to the following equation: -

$$
P_{d g, \min } \leq P_{d g} \geq P_{d g, \max }
$$

Thus, the objective function for this study, in terms of loss is given by: -

$$
\min f=\sum_{i=1}^{a} P_{i}
$$

Where

$i=$ location of DG unit

$P_{i}=$ Nodal injection of power at bus $\mathrm{i}$

$a=$ total bus number

To find the optimal size of DG at random bus, which only supply real power in order to minimize losses

$$
\begin{aligned}
\min f= & \sum_{i=1}^{a} P i+a 1\left(\frac{\text { Ppvinject }}{P p v(\text { level })}\right)+ \\
& a 2\left[\sum_{u v}(V u v-V 1 \max )^{2}+\right. \\
& \left.\sum_{l v}(V l v-V 1 \max )^{2}\right]
\end{aligned}
$$

where

$=$ total PVs injected power

$=$ the $\mathrm{PV}$ maximum power penetration

$=$ the penalty factors

$=$ bus violating the upper voltage limit

= bus violating the lower voltage limit

\section{Methodology}

\subsection{Evolutionary programming}

In this study, a methodology for the optimal location and sizing for DG Type 1 which only inject real power is considered. In this study photovoltaic is chosen as the compensation device optimized using EP. Evolutionary programming was first invented by Dr. Lawrence J. Fogel (1928-2007) while serving at the National Science Foundation in 1960 . He had been tasked to provide a report to the U.S. Congress on the worth of investing in basic research. The EP is a stochastic optimization strategy. In EP; it initially starts with initialization process to generate random number. Mutation process is applied to each individual value based on population to obtain the new individuals and form new individuals called as the offspring. Mutations is an important process to breed the offsprings which affect the behavior of the individual to find the best optimal value. Subsequently, in the combination process; the offspring population is combined with the parent population doubled the number of total individuals. The general flowchart for the EP is shown in Figure 1.

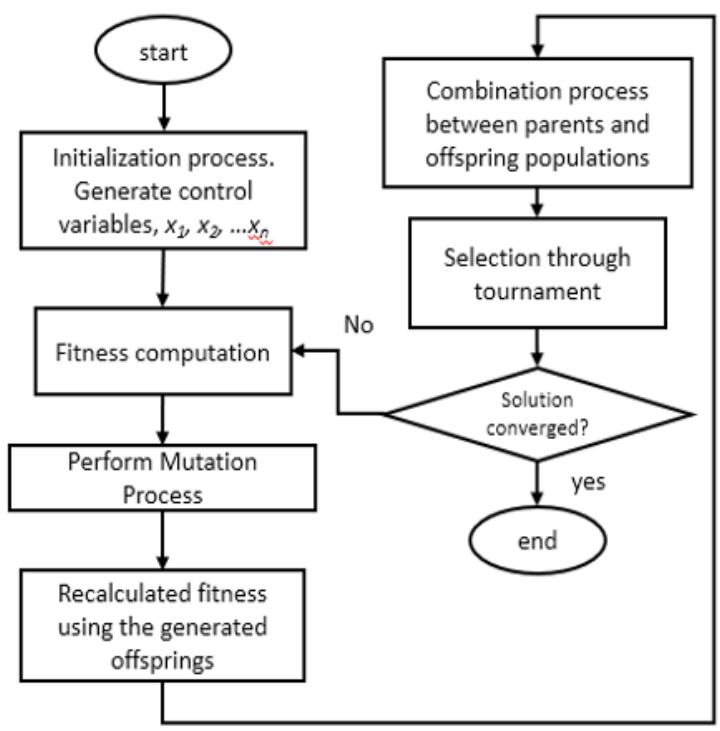

Fig. 1. Flow chart of the EP

\subsection{Random Number Initialization}

In the beginning of the initialization stage, the EP program was written to generate a set of three random numbers, $x_{1}, x_{2}$ and $x_{3}$. These variables represent the optimal bus locations to be installed with three units of PVs. Variables $x_{4}, x_{5}$ and $x_{6}$ represent the optimal size or power to be injected into the system. All of these six variables are the positions of the best fit individuals assigned as parents. The constraints are set during the initialization stage to let the EP only generates random numbers within the required conditions.

No_bus=30; \% This is the load bus value

Bus_value $=5 ; \%$ The bus voltage

Xlocation $=$ randi $\left(x \max ,\left[\begin{array}{ll}1 & D\end{array}\right]\right) \%$ random number

$\%$ represents the location or bus for $D G$ installation.

$X$ sizing $=\operatorname{rand}(x, y) * A+B \%$ The random number to

$\%$ represent the sizing of $D G$

Where

$D=$ number of $D G$

The maximum size of multiple DG is equal to $2 \mathrm{MW}$, which is injected based on the random location in the system. In this case, load $Q_{d}$ had been injected at bus 30 in which bus 30 is the critical bias in this system. In the system, bus 30 have the lower voltage value in the system after completed the load flow. It also has the lowest maximum loadability value. 


\subsection{Fitness Calculation}

Fitness value is calculated for all the individuals in the population. Fitness values are computed twice; one is computed using the individual random values in the parents; while, the second one is calculated using the offsprings. The PV values will be the control variables for the losses minimization or voltage profile improvement in the system. The MATLAB syntax can be written below: -

busdata (bus_no,6)=load_injected

busdata(xlocation, 7$)=x$ sizing

\subsection{Mutation}

The next process is the mutation which produces the offspring from the parent's population based on the Gaussian Mutation equation as below:

$$
X_{i+m, j}=X_{i, j}+\left(0, B\left(X_{j \max }-X_{j \min }\right)\left(\frac{f_{r}}{f \max }\right)\right.
$$

Where

$X_{i+m, j}=$ mutate parent

$X_{i, j}=$ parents

$\beta=$ range of mutation; $0<\beta<1$

$X_{\text {jmax }}=$ maximum random number for every variable

$X_{j m i n}=$ minimum random number for every variable

$f_{r}=$ fitness for $\mathrm{r}^{\text {th }}$ random number

$\mathrm{f}_{\max }=$ maximum fitness

\subsection{Combination}

Combination in EP is a process to combine the parent and offspring populations in cascode which eventually doubled up the number of individuals. The value of parent and offspring determine from the calculation of fitness 1 and fitness 2 . The number of rows will be doubled after combination

\subsection{Selection}

Selection process is conducted to select the best individuals for the next iteration. The membership for this population is the combination of the parent and offspring populations.

\subsection{Convergence Test}

The convergence test is conducted to determine the stopping criterion. It is characterized by the difference between the minimum fitness and maximum fitness $\leq$ 0.0001 . The mathematical equation for the stopping criterion is given by: -

$$
\max _{\text {fitness }}-\min _{\text {fitness }}=\leq 0.0001
$$

\section{Result and Discussion}

The implementation of DG into the system to minimize the loss value and improve voltage profile is conducted on the IEEE 30-Bus RTS. Load increment was subjected to bus 30 in the system, ranging from 5 MVAR to 30 MVAR with the interval of 5 MVAR. Table 1 tabulated the results for the losses variation as the load was increased. In general, the losses in the system increase accordingly as the reactive power loading was increased. 3 DGs were installed in the system for this compensation scheme. For instance, at $20 \mathrm{MVAR}$ reactive loading, the implementation of the EP has managed to reduce the loss of the system from 19.5484 MW to $18.704 \mathrm{MW}$, leading to $4.32 \%$ loss reduction. This requires $1.247 \mathrm{MW}, 1.983$ MW and 1.725 MW to be installed on buses 23, 30 and 11. Results for other loading condition can be referred to the same table. On the other hand, for voltage profile improvement, the implementation of EP managed to improve the voltage from 0.8438 p.u. to 0.8521 p.u. as

Table 1. Total Losses Variation With Respect to Reactive Load increment

\begin{tabular}{ccccccccc}
\hline \multirow{2}{*}{$\begin{array}{c}\text { Load } \\
\text { (MVAR) }\end{array}$} & \multicolumn{3}{c}{ Opt Location } & \multicolumn{3}{c}{ Opt size DG } & \multicolumn{2}{c}{ Total losses } \\
\cline { 2 - 8 } & DG1 & DG2 & DG3 & P1 & P2 & P3 & $\begin{array}{c}\text { Without } \\
\text { DG }\end{array}$ & With DG \\
\hline 0 & 30 & 8 & 26 & 1.694 & 1.455 & 1.817 & 17.5640 & 16.788 \\
5 & 23 & 30 & 11 & 1.247 & 1.983 & 1.724 & 17.7038 & 16.945 \\
10 & 23 & 30 & 11 & 1.247 & 1.983 & 1.725 & 18.1091 & 17.248 \\
15 & 23 & 30 & 11 & 1.247 & 1.983 & 1.724 & 18.6660 & 17.776 \\
$\mathbf{2 0}$ & $\mathbf{2 3}$ & $\mathbf{3 0}$ & $\mathbf{1 1}$ & $\mathbf{1 . 2 4 7}$ & $\mathbf{1 . 9 8 3}$ & $\mathbf{1 . 7 2 5}$ & $\mathbf{1 9 . 5 4 8 4}$ & $\mathbf{1 8 . 7 0 4}$ \\
25 & 23 & 30 & 11 & 1.247 & 1.983 & 1.725 & 20.9265 & 20.012 \\
30 & 23 & 30 & 11 & 1.247 & 1.983 & 1.725 & 23.4419 & 22.356 \\
\hline
\end{tabular}

Table 2. Voltage Variation With Respect to Reactive Load Increment

\begin{tabular}{ccccccccc}
\hline \multirow{2}{*}{$\begin{array}{c}\text { Load } \\
\text { (MVAR) }\end{array}$} & \multicolumn{2}{c}{ Opt Location } & \multicolumn{3}{c}{ Opt size DG } & \multicolumn{2}{c}{$\mathrm{V}_{\mathrm{m}}$} \\
\cline { 2 - 9 } & DG1 & DG2 & DG3 & P1 & P2 & P3 & $\begin{array}{c}\text { Without } \\
\text { DG }\end{array}$ & With DG \\
\hline 5 & 23 & 30 & 11 & 1.247 & 1.982 & 1.725 & 0.9728 & 0.9793 \\
10 & 23 & 30 & 11 & 1.247 & 1.982 & 1.725 & 0.9326 & 0.9421 \\
15 & 23 & 30 & 11 & 1.247 & 1.982 & 1.725 & 0.8910 & 0.9012 \\
20 & 23 & 30 & 11 & 1.247 & 1.983 & 1.725 & 0.8438 & 0.8521 \\
25 & 23 & 30 & 11 & 1.247 & 1.983 & 1.725 & 0.7881 & 0.7978 \\
30 & 23 & 30 & 11 & 1.247 & 1.983 & 1.725 & 0.7069 & 0.7197 \\
\hline
\end{tabular}




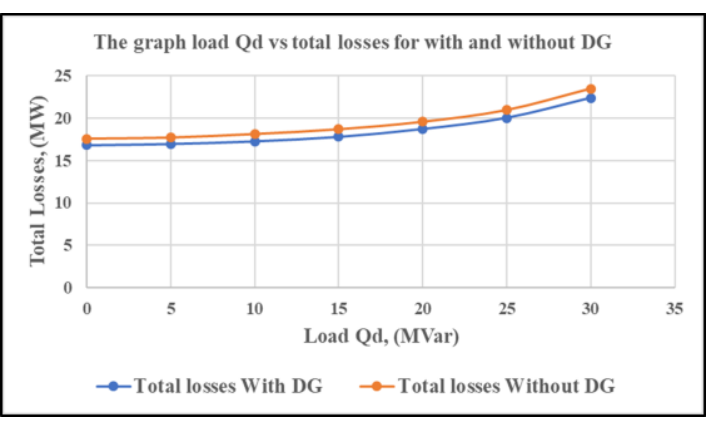

Fig. 2. The graph for load Qd versus total losses with DG and without DG.

tabulated in Table 2. This will require $1.247 \mathrm{MW}, 1.983$ MW and $1.725 \mathrm{MW}$ of DG values to be installed on buses 23, 30 and 11. Further presentation of results can be noticed in Figure 2 and Figure 3. In general, the losses profile increase as the reactive power loading is increased from 5 MVAR to 30 MVAR as shown in Figure 2. The implementation of EP for DG installation has witnessed lower loss profile. This implies that the DG installation scheme is a good option to reduce losses in the system which in turns can be translated into saving in monetary form. On the other hand, Figure 3 illustrates the voltage profile as reactive power loading is increased for both the pre- and post-EP implementation. Pre-EP implementation refers to the results without DG installation; while post-EP refers to the results with the DG installation. In general, with the DG installation, optimized using EP; the voltage profile is higher with the DG installation. The voltage value gradually reduces as the reactive power loading was increased from 5 MVAR to 30 MVAR. This implies that the DG installation, optimized using EP can improve the voltage to significant results. This can also be translated into monetary benefit.

\section{Conclusion}

The installation of DG in the transmission system had reduced the total losses and improved the voltage profile in the system. Results obtained from the study demonstrated that the two objective functions had been achieved for the DG installation scheme as the remedial action or planning in losses and voltage control. Optimal location and optimal sizing of DG had been obtained by using EP. Further study can be planned to utilize the developed EP optimization engine for further implementation either for solving other optimization problems or larger systems or both.

\section{Acknowledgement}

The authors would like to acknowledge the UCSI University for the financial support of this project. This research is supported by UCSI University under the Centre of Excellence for Research, Value Innovation, and Entrepreneurship (CERVIE).

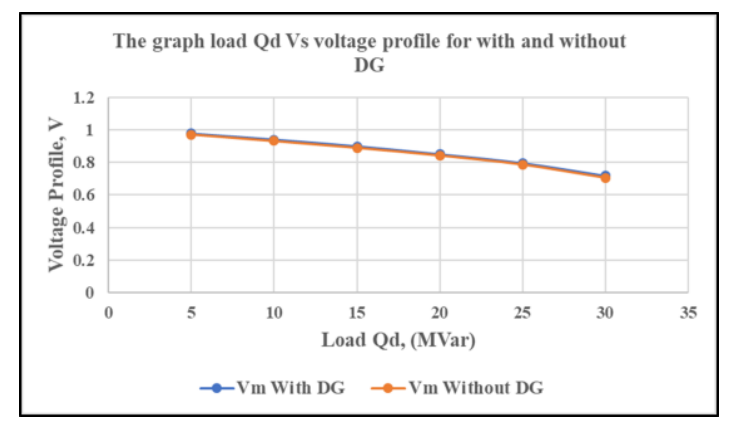

Fig. 3. The graph for load Qd versus voltage profile for with DG and without DG.

\section{References}

1. M. M. Aman, G. B. Jasmon, A. H. A. Bakar, and H. Mokhlis, "A new approach for optimum simultaneous multi-DG distributed generation Units placement and sizing based on maximization of system loadability using HPSO (hybrid particle swarm optimization) algorithm," Energy, vol. 66, pp. 202-215, (2014)

2. T. Ackermann, G. Andersson, and L. Söder, "Distributed generation: A definition," Electr. Power Syst. Res., vol. 57, no. 3, pp. 195-204, (2001)

3. L. Y. Wong, S. R. A. Rahim, M. H. Sulaiman, and O. Aliman, "Distributed generation installation using particle swarm optimization," no. June, pp. 159-163, (2010)

4. S. Ghosh, S. P. Ghoshal, and S. Ghosh, "Optimal sizing and placement of distributed generation in a network system," Int. J. Electr. Power Energy Syst., vol. 32, no. 8, pp. 849-856, (2010)

5. L. I. Dulău, M. Abrudean, and D. Bică, "Distributed Generation Technologies and Optimization," Procedia Technol., vol. 12, pp. 687-692, (2014)

6. Q. Kang, T. Lan, Y. Yan, L. Wang, and Q. Wu, "Group search optimizer based optimal location and capacity of distributed generations," Neurocomputing, vol. 78, no. 1, pp. 55-63, (2012)

7. Rezaee Jordehi, "Allocation of distributed generation units in electric power systems: A review," Renew. Sustain. Energy Rev., vol. 56, pp. 893-905, (2016)

8. J. H. Zhao, J. Foster, Z. Y. Dong, S. Member, and K. P. Wong, "Distributed Generation Impacts," IEEE Trans. Power Syst., vol. 26, no. 3, pp. 1-10, (2010)

9. D. Q. Hung, N. Mithulananthan, and R. C. Bansal, "Analytical strategies for renewable distributed generation integration considering energy loss minimization," Appl. Energy, vol. 105, pp. 75-85, (2013)

10. A. Parizad, A. Khazali, and M. Kalantar, "Optimal placement of distributed generation with sensitivity factors considering voltage stability and losses indices," Proc. - 2010 18th Iran. Conf. Electr. Eng. 
ICEE 2010, pp. 848-855, (2010)

11. N. Khalesi, N. Rezaei, and M. R. Haghifam, "DG allocation with application of dynamic programming for loss reduction and reliability improvement," Int. J. Electr. Power Energy Syst., vol. 33, no. 2, pp. 288-295, (2011)

12. J. J. Jamian, M. W. Mustafa, H. Mokhlis, and M. N. Abdullah, "Comparative study on Distributed Generator sizing using three types of Particle Swarm Optimization," Proc. - 3rd Int. Conf. Intell. Syst. Model. Simulation, ISMS 2012, pp. 131-136, (2012)

13. D. Q. Hung, N. Mithulananthan, and R. C. Bansal, "Analytical expressions for DG allocation in primary distribution networks," IEEE Trans. Energy Convers., vol. 25, no. 3, pp. 814-820, (2010)
14. D. Zhang, Z. Fu, and L. Zhang, "An improved TS algorithm for loss-minimum reconfiguration in large-scale distribution systems," Electr. Power Syst. Res., vol. 77, no. 5-6, pp. 685-694, (2007)

15. T. T. Nguyen, A. V. Truong, and T. A. Phung, "A novel method based on adaptive cuckoo search for optimal network reconfiguration and distributed generation allocation in distribution network," Int. J. Electr. Power Energy Syst., vol. 78, pp. 801-815, (2016)

16. T. G??zel and M. H. Hocaoglu, "An analytical method for the sizing and siting of distributed generators in radial systems," Electr. Power Syst. Res., vol. 79, no. 6, pp. 912-918, (2009) 